\title{
Extended Born-Infeld Dynamics and Cosmology
}

\author{
M. Novello, ${ }^{1}$ M. Makler, ${ }^{1,2,3}$ L. S. Werneck, ${ }^{4}$ and C. A. Romero ${ }^{5}$ \\ ${ }^{1}$ Centro Brasileiro de Pesquisas Físicas, \\ Rua Xavier Sigaud, 150, CEP 22290-180, Rio de Janeiro, RJ, Brazil \\ ${ }^{2}$ Observatório Nacional - MCT, \\ Rua Gal. José Cristino, 77, CEP 20921-400, Rio de Janeiro, RJ, Brazil \\ ${ }^{3}$ Universidade Federal do Rio de Janeiro, Instituto de Física, \\ C.P. 68528, CEP 21941-972, Rio de Janeiro, RJ, Brazil \\ ${ }^{4}$ Universidade Federal do Rio de Janeiro, Observatório do Valongo, \\ Ladeira Pedro Antônio, 43, CEP 20080-090, Rio de Janeiro, RJ, Brazil \\ ${ }^{5}$ Universidade Federal da Paraíba, Departamento de Física, \\ C.P. 5008, CEP 58051-970, João Pessoa, PB, Brazil
}

\begin{abstract}
We introduce an extension of the Born-Infeld action for a scalar field and show that it can act as unifying-dark-matter, providing an explanation for both structure formation and the accelerated expansion of the universe. We investigate the cosmological dynamics of this theory in a particular case, referred as the "Milne-Born-Infeld" (MBI) Lagrangian. We show that this model, whose equation of state has effectively a single free parameter, is consistent with recent type Ia supernovae data, providing a fit as good as for the $\Lambda$ CDM model with the same number of degrees of freedom. Furthermore, this parameter is tightly constrained by current data, making the model easily testable with other observables. Contrary to previous candidates for unifying-dark-matter, the sound velocity of the MBI model is vanishing both close to the dark matter state as well as near the cosmological constant state. This could avoid the problems on the matter power spectrum that were present in previous adiabatic dark-matter/dark-energy unification models. We also present a short discussion on the causal propagation in nonlinear scalar field theories such as the one proposed here.
\end{abstract}

PACS numbers: 98.80.Es, 98.80.-k, 98.80.Cq, 04.20.-q, 11.10.-z

\section{INTRODUCTION}

In the current standard cosmological model, it is often assumed that two unknown components dominate the dynamics of the universe: dark-matter and dark-energy. Dark-matter has been for several decades a fundamental ingredient in cosmological model building. Its need is recognized, for instance, to explain galactic rotation curves, virial motions and $X$-ray gas emission in galaxy clusters, large scale flows, and gravitational lensing, all of which are consequences of its local clustering. This clustered component contributes to roughly a third of the critical density.

On the turn to the $21^{\text {st }}$ century, compelling evidence appeared for a negative pressure component - dubbed dark-energy - capable of producing the accelerated cosmic expansion implied by type Ia supernovae data (SNIa, see ref. [1]) and providing around two thirds of the density needed to explain the almost flatness indicated by cosmic microwave background radiation (CMBR) observations [2].

Now, more evidence has been gathered to support the existence of such a negative pressure component. For example, new SNIa observations extending to higher redshifts [3, 4] indicate that the universe was decelerating in the past, implying that the apparent acceleration cannot be due to simple systematic effects on the data. Independently, constraints on the age of the Universe [5] also support the need of cosmic acceleration. The combination of several cosmological observables, such as CMBR anisotropies [6], the large-scale structure [7], and $X$-ray clusters [8], point to the same conclusion.

These evidences were further strengthened by the reported detection of a late Integrated Sachs-Wolfe effect through the cross correlation of CMBR anisotropies and the galaxy distribution 9$]$. In a nearly flat universe this effect is significative only if some matter component with non negligible relativistic pressure is present.

Thus, assuming the validity of Einstein's Theory of Gravity, most of the pillars of modern cosmology seem to converge to a same conclusion: the universe is filled with a negative pressure component, besides dark-matter. It is nevertheless puzzling that the dynamics of our universe be dominated by two seemingly independent components whose nature is unknown. Although several candidates are available for the dark-matter (such as the neutralino and the axion [10]) and for the dark-energy (such as the cosmological constant or a scalar field [1]]), none has been detected in laboratory experiments. This situation has motivated the search for unifying-dark-matter (UDM) models, where a single component is responsible for both structure formation and the current accelerated expansion of the universe.

One example of UDM is given by the Chaplygin Gas and generalizations thereof 12, 13, 14, 15], which were widely studied in the literature and tested against observational data (see for instance 16] and references therein). The Chaplygin gas can be obtained from the Born-Infeld action for a scalar field and has several physical motivations. Nevertheless, the simplest implementation of this model is in disagreement with large-scale 
structure data [17.

In this work we introduce a new model for dark-energy and dark-matter unification based on an extension of the Born-Infeld Lagrangian. It is shown that this matterenergy component can drive the late time acceleration of the universe while behaving as dark-matter in early epochs and at present in high density regions. We focus on a subclass of such model which we refer as the "MilneBorn-Infeld" (MBI) Lagrangian. We study the dynamics of the MBI model and show that a de Sitter state is an attractor for the solutions of cosmological interest. Moreover, the "sound velocity" $c_{s}=\delta p / \delta \rho$ is close to zero both in the early universe and in the de Sitter epoch. This could solve the problem of instabilities present in previous unifying-dark-matter models 17, 18.

We show that cosmological observables in the MBI model, such as the luminosity distance, depend upon a single effective free parameter $R$ (besides the "standard" cosmological parameters). We use recent data on 194 SNIa to place a strong constraint on $R$. The MBI model provides as a good fit to the data as the $\Lambda$ CDM model with the same number of free parameters (assuming a flat universe).

The paper is organized as follows. We start by reviewing the dynamics of purely kinetic noncanonical Lagrangians for a scalar field and display its equivalence to a perfect fluid description (sec. III). We then discuss the effective geometry for nonlinear scalar field theories (sec. III) and review the Born-Infeld model in light of this approach (sec. IV). In section $\nabla$ we introduce an extension of the Born-Infeld Lagrangian and discuss its role as a unifying-dark-matter candidate. We turn to a particular case of this Lagrangian - the MBI model and derive its corresponding equation of state in [D] The sound speed and equation of state parameter, as well as a comparison to other UDM models, are discussed in section VIII The dynamics of homogeneous solutions of the MBI model is studied in section VIII In section IX] it is explicitly shown that this model has a late time accelerating phase. The Hubble parameter as a function of the scale factor is derived in $\mathbf{X}$ and the results are applied to type Ia supernovae data in section XI We sum up the results and present our concluding remarks in section XII Some speculations about the possible role of the proposed model in the early universe are discussed in the appendix.

\section{KINETIC LAGRANGIAN AND THE PERFECT FLUID REPRESENTATION}

In this work we shall consider scalar field models whose Lagrangian have a noncanonical form

$$
L=V(\varphi) F(W),
$$

where $W:=\nabla_{\mu} \varphi \nabla^{\mu} \varphi$.

Noncanonical (i.e., non-quadratic) kinetic terms appear rather frequently in low-energy effective La- grangians, generated by radiative corrections in the underlying true theory (see e.g. [19]). For example, in string and supergravity theories, nonlinear kinetic terms arise generically in the effective action describing moduli and massless degrees of freedom [20]. Also, scalar fields are common many particle physics theories beyond the standard model. It is therefore not surprising that scalar fields with noncanonical kinetic terms could play a relevant role in an effective description of nature. If we expect the equations of motion in classical theories to be of second order, it is natural to consider only kinetic terms which are functions of the square of the gradient of the scalar field 21]. If it is further assumed that the Lagrangian is factorizable, such as in tachyon condensates [22], we are led to expression (11). This type of Lagrangian also appears as an effective theory for the low energy expansion of fluctuations around a ghost condensate (neglecting terms involving more than one derivative of $\varphi$, see e.g. [23]).

Lagrangians of the form (11) were first introduced in the cosmological setting in the context of inflation 24, 25], then as a dark-energy candidate [26], and finally as UDM candidates, such as in the tachyon [27] and ghost condensate 23] models for dark-matter and dark-energy unification. ${ }^{1}$

For simplicity, throughout this work we shall consider Lagrangians with constant potentials, i.e. of the type

$$
L=L(W) \text {. }
$$

Such models were the first ones investigated in the inflationary setting [24]. To this family belongs the BornInfeld action, which leads to the Chaplygin gas model. Some general properties of this type of Lagrangian in the context of UDM are discussed in refs. 21, 29].

Before closing this section let us display the equivalence of Lagrangian (2) to a perfect fluid description. The standard definition of the energy-momentum tensor is given by

$$
T_{\mu \nu}=\frac{2}{\sqrt{-g}} \frac{\delta(\sqrt{-g} L)}{\delta g_{\mu \nu}},
$$

which, in the case of the scalar field with a purely kinetic Lagrangian (2), leads to

$$
T_{\mu \nu}=-L g_{\mu \nu}+2 L_{W} \nabla_{\mu} \varphi \nabla_{\nu} \varphi,
$$

where $L_{W}:=\partial L / \partial W$. As is well-known, any energymomentum tensor can be written in terms of a fluid by the choice of a particular frame represented by an observer endowed with a four-velocity field $v^{\mu}$, yielding the decomposition

$$
T_{\mu \nu}=\rho v_{\mu} v_{\nu}-p h_{\mu \nu}+q_{(\mu} v_{\nu)}+\pi_{\mu \nu},
$$

\footnotetext{
1 It is worth mentioning that other nonlinear theories, such as for spin 1 fields, may also play an important role in cosmology (see, e.g., ref. [28] and refs. therein).
} 
where the ten independent quantities $\rho, p, q^{\alpha}$ and $\pi_{\alpha \beta}$ are obtained through the projections of $T_{\mu \nu}$ onto $v^{\alpha}$ and the space orthogonal to it, and $\pi_{\alpha \beta}$ is traceless. ${ }^{2}$

In the frame commoving with the gradient of the field, defined by the normalized vector

$$
v_{\mu}:=\frac{\nabla_{\mu} \varphi}{\sqrt{\|W\|}},
$$

the energy-momentum tensor (4) is equivalent to that of a perfect fluid, since the heat flux $q_{\alpha}$ and the anisotropic pressure $\pi_{\mu \nu}$ vanish identically. In this case, the non identically zero quantities are the energy density and pressure, given by:

$$
\rho=-L+2 L_{W} W, \quad p=L .
$$

Moreover, since both $p$ and $\rho$ are given as a function of $W$ only, one of these relations can be inverted to provide $p=p(\rho)$. We see therefore that there can be no bulk viscosity. This shows that the dynamics given by any purely kinetic Lagrangian is equivalent to that of a perfect fluid, both in its energy-momentum tensor and equation of state (eos).

\section{CAUSALITY AND EFFECTIVE GEOMETRY}

Motivated by the recent interest on nonlinear scalar field theories in the cosmological context 21, 23, 24, 25, 26, 27, 29], we find it useful to present a brief discussion on the issue of the causal propagation of the field. Our purpose is to study the behavior of discontinuities of the equations of motion around a fixed background solution. Instead of using the traditional perturbation method (the eikonal approximation), we shall use a more elegant formalism synthesized in the work of Hadamard [30]. In this method, the propagation of high-energy perturbations of the field is studied by following the evolution of the wave front, through which the field is continuous but its first derivative is not. To be specific, let $\sigma$ be the surface of discontinuity defined by the equation

$$
\sigma\left(x^{\mu}\right)=\text { const } .
$$

The discontinuity of a function $J$ through the surface $\sigma$ will be represented by $[J]_{\sigma}$, and its definition is

$$
[J]_{\sigma}:=\lim _{\delta \rightarrow 0^{+}}\left(\left.J\right|_{\sigma+\delta}-\left.J\right|_{\sigma-\delta}\right) .
$$

The discontinuities of the field and its first derivative are given by

$$
[\varphi]_{\sigma}=0, \quad\left[\nabla_{\mu} \varphi\right]_{\sigma}=0
$$

\footnotetext{
${ }^{2}$ Explicitly, the scalars $\rho$ and $p$ (energy density and pressure) are defined by $\rho:=T^{\alpha \beta} v_{\alpha} v_{\beta}$ and $p:=-\frac{1}{3} h_{\alpha \beta} T^{\alpha \beta}$, the heat flux is $q^{\alpha}:=h^{\alpha \beta} v^{\gamma} T_{\beta \gamma}$, and the traceless symmetric anisotropic tension is $\pi^{\alpha \beta}:=h^{\alpha \mu} h^{\beta \nu} T_{\mu \nu}+p h^{\alpha \beta}$, where $h_{\mu \nu}=g_{\mu \nu}-v_{\mu} v_{\nu}$.
}

while for the second derivative we have

$$
\left[\nabla_{\mu} \nabla_{\nu} \varphi\right]_{\sigma}=\chi k_{\mu} k_{\nu} .
$$

where the vector $k_{\mu}$ is the normal to the surface of discontinuity. Using these values in the equation of motion for the field $\varphi$,

$$
\nabla_{\mu}\left(L_{W} \nabla^{\mu} \varphi\right)=0
$$

we obtain

$$
k_{\mu} k_{\nu} g_{e f f}^{\mu \nu}=0,
$$

where the effective metric is given by

$$
g_{\text {eff }}^{\mu \nu}=L_{W} g^{\mu \nu}+2 L_{W W} \nabla^{\mu} \varphi \nabla^{\nu} \varphi
$$

and $g^{\mu \nu}$ is the background metric. Only in the case of a linear theory $L=W$, the metric that controls the propagation of the discontinuities of the field coincides with the background metric.

Therefore, the propagation of discontinuities of the scalar field, which we shall refer as scalaron, follows null curves in an effective metric that is not universal, but instead depends on the field configuration. We should emphasize that this property is quite generic for any nonlinear field theory (see for instance refs. 31, 32, 33, 34] and references therein).

In terms of the background geometry we can re-write the equation of propagation as

$$
k_{\mu} k_{\nu} g^{\mu \nu}=-\frac{2 L_{W W}}{L_{W}}\left(k_{\mu} \nabla^{\mu} \varphi\right)^{2} .
$$

This means that in the background geometry the scalaron behaves as time-like particles in cases in which $L_{W} L_{W W}<0$, and it behaves as tachyons in the cases in which $L_{W} L_{W W}>0$. We shall came back to this issue in the appendix.

\section{EXCEPTIONAL LAGRANGIAN: THE BORN-INFELD CASE}

The effective metric is an auxiliary instrument to simplify the description of the propagation of the discontinuities of nonlinear field theories. In nonlinear dynamics it does not correspond to the background geometry in which the field acts. From this property one could conclude that such geometry should not appear in any aspect of the dynamics of the field. This is indeed the case in the most common theories, however, in certain nonlinear theories the dynamics is described in terms of quantities associated to the effective metric. For example, in Electrodynamics there is at least one exceptional case which is worth to point out: the nonlinear field theory proposed by Born and Infeld [35]. In that theory, the Lagrangian is written in terms of the determinant of the effective metric $L=\left(-\operatorname{det} g_{e f f}\right)^{1 / 4}$ [36]. We shall show below 
that a similar situation occurs for the nonlinear scalar field theory. In order to describe such special dynamics we must face the question: what is the property of the Lagrangian $L(W)$ such that its corresponding dynamics is given only in terms of the effective metric? We shall follow the same lines as for the case of Electrodynamics 32 .

We note that the (inverse) covariant metric tensor of the effective metric (8), defined by the relation $g_{\mu \nu}^{e f f} g_{e f f}^{\nu \lambda}=\delta_{\mu}^{\lambda}$, is given by

$$
g_{\mu \nu}^{e f f}=\frac{1}{L_{W}} g_{\mu \nu}-\frac{2 L_{W W}}{L_{W}\left(L_{W}+2 W L_{W W}\right)} \nabla_{\mu} \varphi \nabla_{\nu} \varphi .
$$

A straightforward calculation shows that the evaluation of the determinant of the effective metric yields the result:

$$
\operatorname{det} g_{e f f}^{\mu \nu}=L_{W}^{3}\left(L_{W}+2 W L_{W W}\right) .
$$

It then follows that the unique theory that can be written in terms of its associated effective metric is the one provided by the Born-Infeld like form:

$$
L_{B I}=-\sqrt{b W+e},
$$

where $b$ and $e$ are constants. Indeed, using (12) into the expression of the determinant (11) we obtain

$$
L_{B I}=\text { const. }\left(\operatorname{det} g_{\mu \nu}^{e f f}\right)^{1 / 6} \text {. }
$$

The energy density and pressure of the effective fluid description for the Born-Infeld dynamics (from eqs. 12 and 5]

$$
\rho=e / \sqrt{b W+e}, \quad p=-\sqrt{b W+e},
$$

are such that the equation of state takes a very simple form $^{3}$

$$
p=-\frac{e}{\rho}
$$

A fluid with the above eos is known as Chaplygin gas ${ }^{4}$ 37 .

The Born-Infeld action for a scalar field was first introduced by Heisenberg in 1939, in the context of meson theory [38]. This model reappeared in the string theory setting, where the Born-Infeld Lagrangian is associated to the parametrization invariant Nambu-Goto $d$-brane action in a $(d+1,1)$ space-time [39]. The

\footnotetext{
3 It is worth noting that, although Lagrangian (12) has two free parameters, only one appears in the eos.

4 This name, which became popular in the $d$-brane setting, is off course inspired on the eos proposed by Chaplygin in 1904. In the original expression, $\rho$ is only the internal energy, not the total relativistic one, and the weak energy condition is not violated, as opposed to what is needed in the cosmological case.
}

Chaplygin gas has recently attracted much interest in the cosmological framework, both as a candidate for dark-energy [12, 40] as well as for unifying-dark-matter 14, 42]. In fact, as can be seen from (15) it has a negative pressure that could power the accelerated expansion of the universe. Also, the pressure is negligible for high density regions and "mechanical" perturbations are stable $\left(d p / d \rho=c_{s}^{2}>0\right)$. Therefore this fluid could also be responsible for structure formation. Because of these properties and its simplicity, equation (15) arises naturally as a toy model allowing dark-energy and dark-matter unification [13]. The behavior of the Chaplygin gas, and simple generalizations thereof, as unifying-dark-matter (or simply quartessence ${ }^{5}$ ) was extensively discussed in the literature (see, for instance, refs. 13, 14, 15, 16, 17, 41, 42, 43, 44, 45, 46] and references therein).

\section{EXTENDED BORN-INFELD LAGRANGIAN}

As pointed out in the previous section, the Born-Infeld Lagrangian arises rather naturally in the context of a geometric description for nonlinear scalar fields. Moreover, as mentioned above, besides its formal appeal, this theory has an interesting behavior in the cosmological context. In fact, eos (15) has provided the first model of dark-matter and dark-energy unification [13, 14]. Nevertheless, this model is ruled out by observations of weak gravitational lensing combined with the large-scale galaxy power spectrum [17] and by the anisotropies on the CMBR 43]. Besides, it is also disfavored by a combination of observational data on the background evolution of the universe [16, 46].

A generalization of the Born-Infeld Lagrangian was proposed in 15 and leads to the so-called Generalized Chaplygin gas 14]. However, this model suffers from the same problems as the Chaplygin gas itself [17, 43].

Nevertheless, the successful aspects of the Born-Infeld model as a candidate for dark-energy and dark-matter unification motivate the search for other models that still retain some of its simplicity at the same time avoiding its shortcomings. As shall be discussed in details in the forthcoming sections, such a model could be given by the Extended Born-Infeld (hereafter EBI) Lagrangian represented by

$$
L=-\sqrt{a W^{2}+b W+e} .
$$

The original idea of Born and Infeld [35] was to deal with a theory that describes the evolution of electromagnetic fields that are bounded from above. Here we ap-

\footnotetext{
5 As in this scenario there is a single extra component besides "baryons", neutrinos, and radiation (as opposed to having both dark-matter and dark-energy), the unifying-dark-matter is dubbed as quartessence [4].
} 
ply such limitation idea to the case of a scalar field, as in Lagrangian (12). However, instead of having only a maximum value we also require the existence of a minimum (such that $a<0$ and $b^{2}>-4 a e$ ). Therefore, $W$ is defined between the two roots of the polynomial in (16). From Lagrangian (16), the energy density and pressure of the effective fluid are given by

$$
\begin{aligned}
& \rho=-L+\frac{W(b+2 a W)}{L}, \\
& p=L .
\end{aligned}
$$

The equation of motion (6) derived from Lagrangian (16) is

$$
\nabla_{\mu}\left(\frac{(b+2 a W)}{L} \nabla^{\mu} \varphi\right)=0 .
$$

Therefore, a particular solution for the dynamics of the scalar field is given by a constant value of $W$, namely

$$
W_{0}=-\frac{b}{2 a} \text {. }
$$

In this case, the functional derivative of the Lagrangian $L_{W}$ vanishes and consequently the tensor $T_{\mu \nu}$ becomes identical to the energy distribution of a cosmological constant. We shall call such a configuration the fundamental state. As we shall see later on, in the cosmological case there exists two de Sitter states associated with such constant $W_{0}$, one which is stable and the other unstable. Equation (19) allows also the trivial solution $\varphi=$ const. which corresponds to another "vacuum" state. We shall see latter that, at least in the cosmological case, the physically relevant vacuum is given by the fundamental state (20).

Notice that, near the roots of the quadratic function, $L \simeq 0$ and the scalar field acts as pressureless matter. Besides, close to at least one of these roots the density is positive. Thus we have the possibility of a matter-energy component that interpolates between dust (close to a root of [16) and a cosmological constant like configuration (at the fundamental state). Moreover, the presence of $L$ in the denominator in expression (17) shows that the scalar field behaves as dust in high density regions. This situation is typical from unifying-dark-matter models, where this component acts as dark-matter in clustered regions and as dark-energy in low density regions, providing and explanation for the accelerated expansion of the universe [13. We shall investigate this model in the cosmological setting in the forthcoming sections. Our analysis will be focused on a special case of Lagrangian (16) as described below.

\section{THE MILNE-BORN-INFELD LAGRANGIAN}

In the reminder of this article we shall restrict ourselves to a subclass of Lagrangian (16), namely the case in which $e=0$. In this situation one root corresponds to a $p=\rho=0$ state and there is a positive root (we choose $b>0)$ corresponding to a dust limit. As we shall see in section VIII this model allows a solution representing an expanding empty $(\rho=0)$ universe, which is known as the Milne solution. For this reason we refer to this model as the "Milne-Born-Infeld" (hereafter MBI) Lagrangian. In this case it is convenient to express (16) as

$$
L=-A \sqrt{W\left(\Sigma^{2}-W\right)},
$$

where $A:=\sqrt{|a|}$ and $\Sigma^{2}:=b A^{-2}$. For this model, the whole admissible spectra of values for $W$ is contained in the domain $\left(0, \Sigma^{2}\right)$. The fundamental state is located at $\Sigma^{2} / 2$.

In the MBI model, the energy density and pressure (eqs. 17 and 18) are given by

$$
\begin{aligned}
& \rho=\frac{A W^{2}}{\sqrt{W\left(\Sigma^{2}-W\right)}}, \\
& p=-A \sqrt{W\left(\Sigma^{2}-W\right)} .
\end{aligned}
$$

Here the expression of the eos $p(\rho)$ is rather complicated, as opposed to the Born-Infeld case (eq. 15). It is more convenient to write $\rho$ as a function of $p$ :

$$
\rho=\left(A \Sigma^{2}+\sqrt{A^{2} \Sigma^{4}-4 p^{2}}\right)^{2} / 4 p .
$$

It is easy to see that, for high densities $\left(\rho \gg A \Sigma^{2}\right)$, the equation of state goes asymptotically to the Chaplygin one

$$
p \simeq-\frac{A^{2} \Sigma^{4}}{\rho} .
$$

The density and pressure of the fundamental state are given by

$$
\rho_{\mathrm{v}}:=\frac{A \Sigma^{2}}{2}=-p_{\mathrm{v}} .
$$

In figure 1 we show $p$ as a function of $\rho$ in the interval $\Sigma^{2}>W>\Sigma^{2} / 2$, from the dust to the fundamental state. As shall be shown in section VIII this is the relevant interval for cosmology.

Before exploring the dynamics given by the MBI Lagrangian (21), let us point out a fundamental difference with respect to the Born-Infeld case and the ExtendedBorn-Infeld with $e \neq 0$. In these two models, the Lagrangian has a canonical form for small values of $W$ $(W \ll e / b$ and $W \ll b / a)$. However, the MBI model does not have such a canonical limit. Nevertheless, we shall see that the MBI Lagrangian provides an interesting model for the dark sector of the universe.

\section{SOUND SPEED AND EQUATION OF STATE PARAMETER}

In the fluid interpretation, the important quantity that controls the level of stability of perturbations is the 


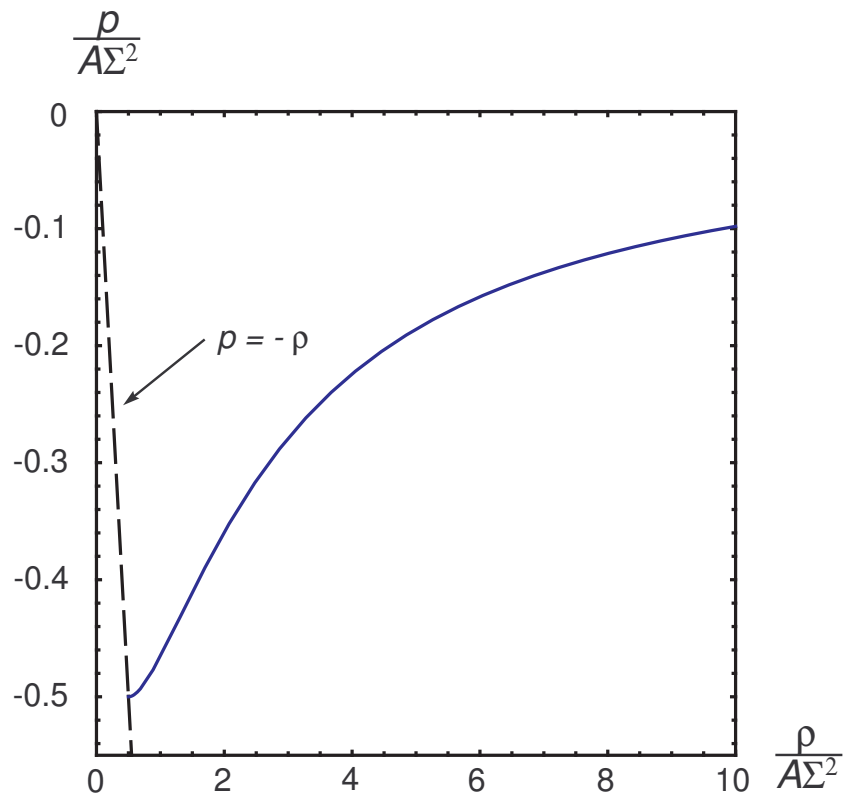

FIG. 1: Equation of state of the MBI model. The dimensionless pressure $p /\left(A \Sigma^{2}\right)$, is plotted as a function of $\rho /\left(A \Sigma^{2}\right)$ starting from the fundamental state $\rho_{\mathrm{v}}=A \Sigma^{2} / 2$. Also displayed is the "vacuum" eos (dashed curve).

"sound velocity" squared $c_{s}^{2}=(\partial p / \partial W) /(\partial \rho / \partial W)$ [25], which in the MBI model is given by

$$
c_{s}^{2}=\frac{2 W^{2}-3 \Sigma^{2} W+\Sigma^{4}}{2 W^{2}-3 \Sigma^{2} W} .
$$

In the interval $\Sigma^{2} / 2<W<\Sigma^{2}$ (from the dust to the de Sitter state) it is immediate to see that the quantity $c_{s}^{2}$ is always positive. Its maximum is given by $c_{s \max }^{2}=1 / 9$ and occurs at $W_{a}=3 \Sigma^{2} / 4$, the value at which the scalar field's self gravity starts being repulsive (see section [X]). For $W<\Sigma^{2} / 2, c_{s}^{2}<0$ and the fluid is mechanically unstable. However, as we shall see in section VIII this is outside the physical domain and such values are never attained in the cosmological framework.

In figure 2 we plot the sound velocity as a function of the density starting at the fundamental state (24). It is interesting to point out some features of the MBI model regarding the sound velocity. First, its maximum value is a third of the velocity of light, whereas in the BornInfeld model it is equal to $c$. Most importantly, near the de Sitter state the sound velocity is zero. As shall be discussed later, our universe is currently approaching this de Sitter state, thus one might expect the present scalaron sound velocity to be small. This is in contrast to most quartessence models studied so far in the literature, where the maximum sound velocity is attained precisely at the de Sitter state [18]. The relatively large value of the sound velocity at present produces strong oscillations and power suppression in the matter spectrum, which render these models incompatible with cur-

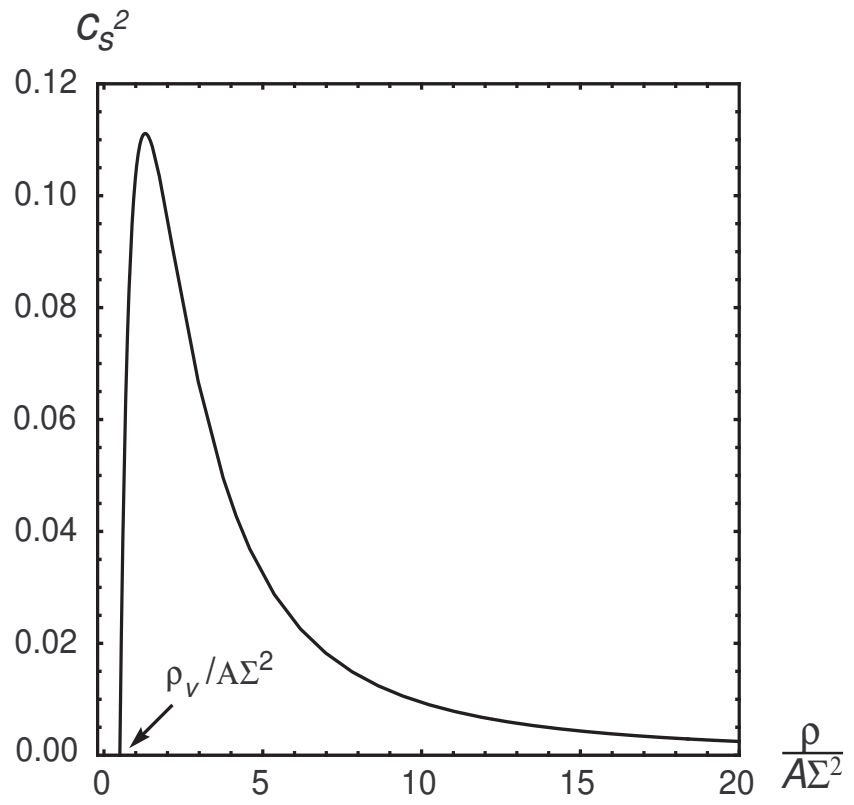

FIG. 2: The sound velocity squared as a function of the dimensionless density $\rho /\left(A \Sigma^{2}\right)$ in the MBI model.

rent data ${ }^{6}$ 17, 18]. The MBI model could avoid this problem since the sound speed is significative only during some limited interval of densities, as shown in the figure.

It is useful to see how the sound velocity $c_{s}^{2}$ evolves as a function of $w:=p / \rho$, which is sometimes know as the "equation of state parameter". Both $c_{s}^{2}$ and $w$ are the quantities that appear in the equations for the evolution of perturbations in the fluid representation (see e.g. ref. [48]). The $c_{s}^{2} \times w$ relation allows also to make a comparison with other quartessence models.

In the MBI case we have $w=1-\Sigma^{2} / W$, such that

$$
c_{s}^{2}=\frac{w(1+w)}{3 w-1} .
$$

In the Born-Infeld model, we have $c_{\mathrm{s}}^{2}=-w$, while for the Generalized Chaplygin gas [12, 14, 15, 41] the relation is $c_{s}^{2}=-\alpha w$. Recently, another generalization of the BornInfeld action was proposed, the $n^{\text {th }}$ order Dirac-BornInfeld theory, given by $L \propto-(1-W)^{1 / n}$ 49], in which case $c_{s}^{2}=-2 w /(n+(n-2)(1+2 w))$. Other two models of quartessence were investigated in [18, 45], where the equations of state are given by $p=-M^{4} \exp \left(-\alpha \rho / M^{4}\right)$ and $p=-M^{4} /\left[\log \left(\rho / M^{4}\right)\right]^{a}$. Here we assume adiabatic perturbations of these models such that $c_{s}^{2}=d p / d \rho$.

\footnotetext{
${ }^{6}$ A particular kind of entropy perturbations solves this problem 18 ,44 but, as discussed in section $\amalg$ models with purely kinetic Lagrangians for scalar fields are adiabatic. Even other scalar field models which have the same background evolution as the Chaplygin gas seem to be highly disfavored [47].
} 

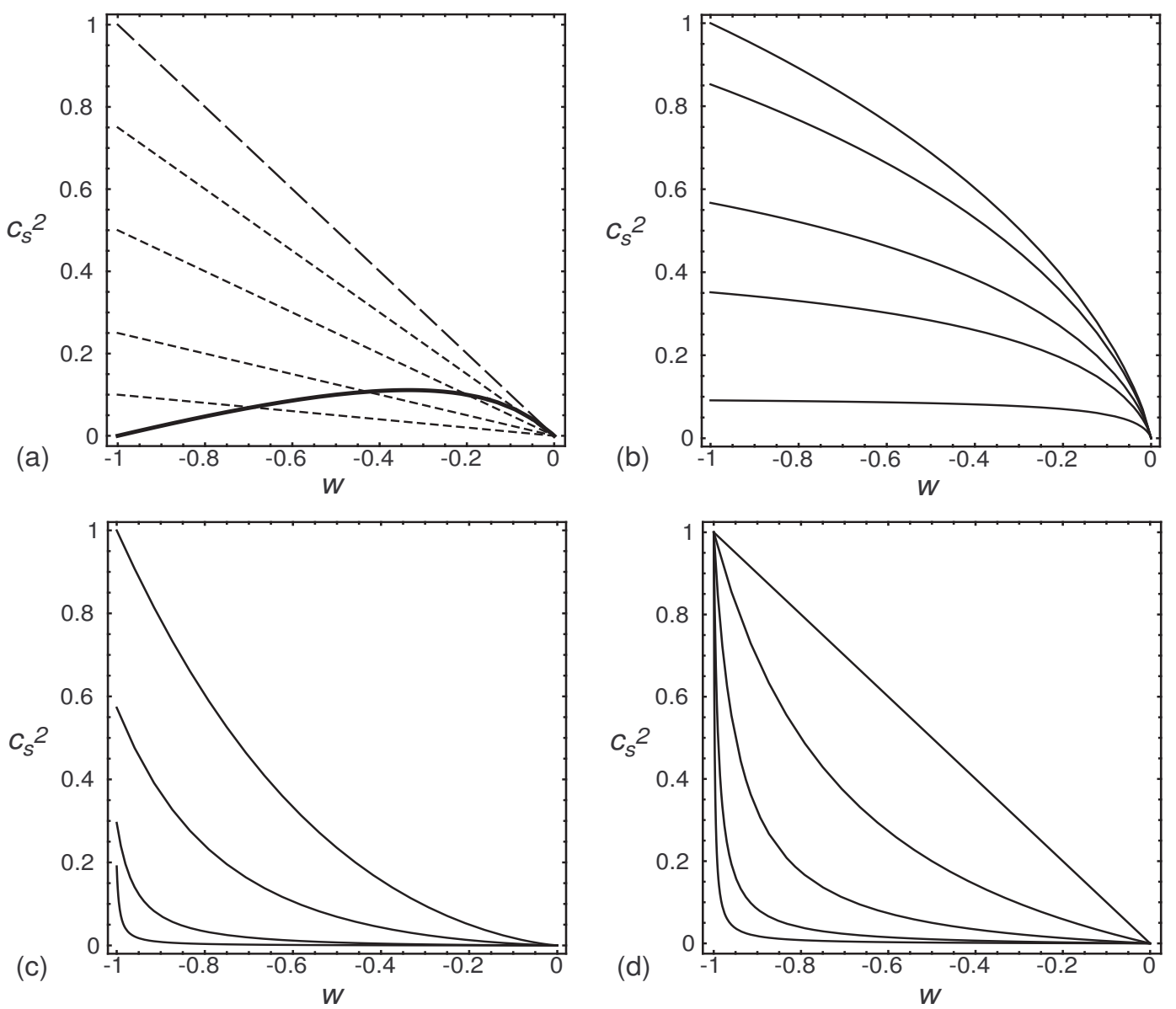

FIG. 3: The sound velocity $c_{s}^{2}$ as a function of the equation of state parameter $w$ for the MBI case and other quartessence models. (a) Results for the MBI model (thick line) and the Generalized Chaplygin gas, with $\alpha=0.1,0.25,0.5$, and 0.75 (from bottom to top, short dashed curves), and $\alpha=1$ (equivalent to the Born-Infeld model, long dashed line). (b) Exponential quartessence with $\alpha=0.1,0.5,1,2$, and $e$ (from bottom to top). (c) Logarithmic quartessence with $\alpha=10^{-3}, 10^{-2}, 10^{-1}$, and $e^{-1}$ (from bottom to top). (d) Results for the $n^{\text {th }}$ order Dirac-Born-Infeld theory with $n=2,5,20,100$, and 500 (from top to bottom).

In figure 3 we plot $c_{s}^{2}$ as a function of $w$ for the models referred above. In the upper left panel we show the results for the MBI (thick line), Born-Infeld (long dashed line), and the Generalized Chaplygin gas models (short dashed curves). In the upper right, the results for the exponential quartessence are displayed. In the lower left panel we plot curves for the logarithmic quartessence model. Finally, in the lower right panel of this figure we show curves for the $n^{\text {th }}$ order Dirac-Born-Infeld theory. From these figures, it is clear that all the models start with $c_{s}^{2}=0$ at $w=0$, where quartessence acts as CDM. Nevertheless, in the MBI case, the sound velocity vanishes again when $w=-1$, whereas in the other models displayed in the figure the sound velocity attains a maximum at that point. This leads to oscillations and suppression in the power spectrum of density perturba- tions. ${ }^{7}$ Thus we expect that this issue of the power spectrum will be alleviated in the MBI model.

\section{DYNAMICS OF SPATIALLY HOMOGENEOUS SOLUTIONS}

Now, let us focus on the dynamics of the MBI model for spatially homogeneous and isotropic configurations.

\footnotetext{
7 This was shown explicitly for the Generalized Chaplygin Gas 17 and the Exponential and Logarithmic models [18]. The behavior of perturbations was not yet studied in the $n^{\text {th }}$ order DiracBorn-Infeld theory [49].
} 
In this case the Raychaudhuri equation is given by ${ }^{8}$

$$
\dot{\theta}+\frac{1}{3} \theta^{2}+\frac{1}{2}(\rho+3 p)=0,
$$

the energy conservation equation is

$$
\dot{\rho}+(\rho+p) \theta=0,
$$

and the expansion parameter can be written as $\theta=3 \dot{a} / a$, where $a(t)$ is the scale factor.

Combining equations (26) and (27) we obtain a dynamical system:

$$
\frac{d \rho}{d t}=F(\rho, \theta), \quad \frac{d \theta}{d t}=G(\rho, \theta),
$$

with

$F=-\theta \frac{A W\left(2 W-\Sigma^{2}\right)}{\sqrt{W\left(\Sigma^{2}-W\right)}}, G=-\frac{1}{3} \theta^{2}-\frac{1}{2} \frac{A W\left(4 W-3 \Sigma^{2}\right)}{\sqrt{W\left(\Sigma^{2}-W\right)}}$,

where, from eq. (22), $W$ is given by

$$
W=\frac{2^{-1 / 3}\left(3 A \Sigma^{2} \rho^{2}\right)^{2 / 3}\left(1+\sqrt{1+\frac{4 \rho^{2}}{27 A^{2} \Sigma^{4}}}\right)^{2 / 3}-(2 / 3)^{1 / 3} \rho^{2}}{\left(9 A^{4} \Sigma^{2} \rho^{2}\right)^{1 / 3}\left(1+\sqrt{1+\frac{4 \rho^{2}}{27 A^{2} \Sigma^{4}}}\right)^{1 / 3}}
$$

From an inspection of (26 27) it is easy to find the four stationary points $P(\rho, \theta)$ of this system. Two correspond to the fundamental state $W=\Sigma^{2} / 2$,

$$
M=\left(\rho_{\mathrm{v}}, \sqrt{3 \rho_{\mathrm{v}}}\right), \quad N=\left(\rho_{\mathrm{v}},-\sqrt{3 \rho_{\mathrm{v}}}\right),
$$

and two are on the line $\theta=0$,

$$
O=(0,0), \quad Q=\left(\rho_{c}, 0\right),
$$

where $\rho_{\mathrm{v}}$ is given by (24) and $\rho_{c}=(3 \sqrt{3} / 2) \rho_{\mathrm{v}}$. Consideration of the behavior of the system in the neighborhood of these points shows that $M$ and $N$ are two-tangent nodes (one stable and the other unstable) while those on the $\theta=0$ line are saddle points.

In figure 4 we display the phase diagram in the $(\rho, \theta)$ plane for equations (28 30), as well as some trajectories. From expression (22) we see that only positive energydensity solutions are allowed. From the figure, it is clear that any trajectory that starts with a density lower than the fundamental state will remain with $\rho \leq \rho_{\mathrm{v}}$. Conversely, trajectories starting at $\rho>\rho_{\mathrm{v}}$ are bounded to have $\rho \geq \rho_{\mathrm{v}}$. It is easy to see from the Friedmann equation that the solutions for the flat case $(k=0)$ give $\rho=\theta^{2} / 3$, which is the dashed trajectory plotted in this

\footnotetext{
${ }^{8}$ For simplicity, in the following sections we shall consider a universe composed only of scalaron matter (baryons will be included in sections $[\mathbf{X}$ and $[\mathrm{XI}$. We choose units such that $8 \pi G=1$.
}

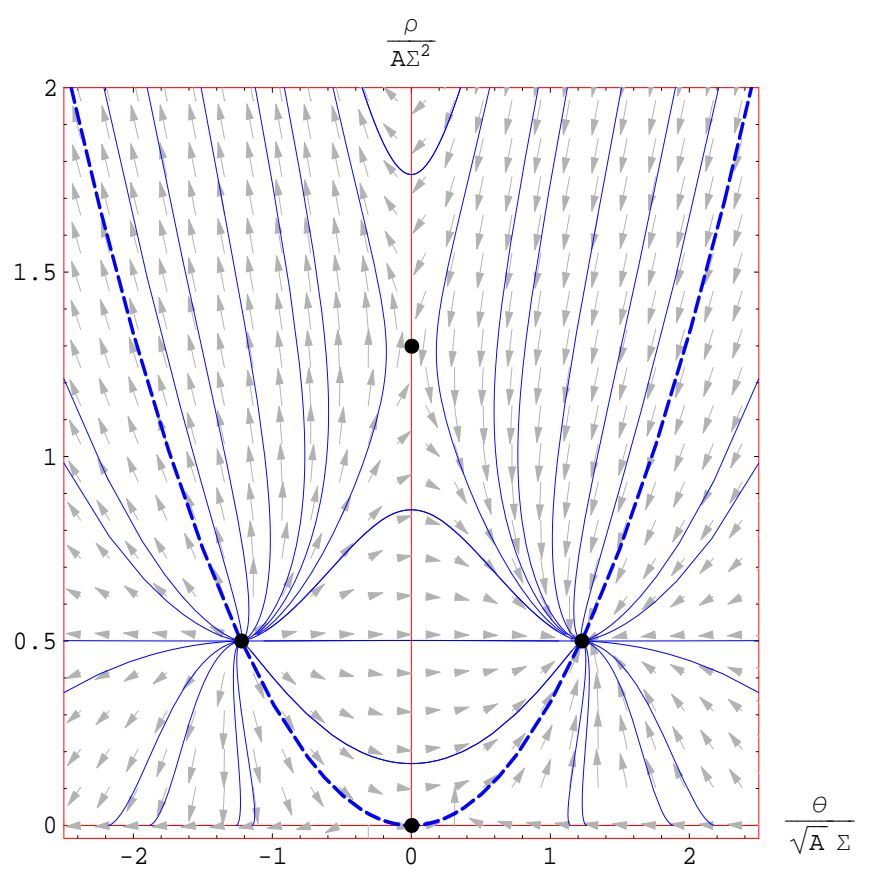

FIG. 4: Vector field and trajectories of the MBI model in the $(\rho, \theta)$ plane. The dashed curve is the trajectory for a flat universe. The dots correspond to the four stationary points $M, N, Q$, and $O$ discussed in the text.

figure. Notice the existence of a trajectory with vanishing density and nonzero expansion (the bottom horizontal line). This is the well known Milne solution, and is allowed only if we have $e=0$ in Lagrangian (16), leading to our nomenclature for this model.

From now on, we shall be concerned with the right hand side of figure 4 i.e., with expanding solutions. Some of these may correspond to the background (unperturbed) cosmological solution, in particular those starting from high values of $\rho$. As a nearly flat spatial geometry is favored by current astrophysical observations, one should only consider trajectories near the $k=0$ one. From figure 4 it is clear that these trajectories end up in the fundamental state $M$. In other words, the de Sitter solution is an attractor for the trajectories of cosmological interest.

It is important to notice that, even in the inhomogeneous case, there is a "vacuum barrier" that cannot be transposed in any expanding region. This can be seen from the energy conservation equation (27), which is valid for any $\theta$. As the vacuum state $p=-\rho$ is approached, the density freezes out, and this is a stable point (the contrary occurs in a contracting region). In the MBI model, only states that initially violate the weak energy condition are able to approach the point $O$, which represents a "static vacuum" ( $\varphi=$ const. $)$. Therefore, as pointed out before, only the domain between the dust regime $W \rightarrow \Sigma^{2}$ and the fundamental state $W_{0}=\Sigma^{2} / 2$ 
— which represents a "dynamical vacuum" — is relevant for the cosmological model. We shall see below that, within this interval, the universe has an accelerated expansion phase.

\section{THE ACCELERATION OF THE UNIVERSE}

The acceleration of the universe is controlled not only by the energy density but also by the pressure, according to the Raychaudhuri equation (26). Writing this equation in terms of the scale factor $a$ and using equations (22) and (23) yields:

$$
\frac{\ddot{a}}{a}=\frac{A W\left(4 W-3 \Sigma^{2}\right)}{\sqrt{W\left(\Sigma^{2}-W\right)}} .
$$

Starting with $W$ close to $\Sigma^{2}$, that is, a matter dominated universe, it is clear that the acceleration changes sign at

$$
W_{a}=\frac{3}{4} \Sigma^{2} .
$$

If the scalaron is the dominant component of the cosmological energy density, the universe will begin to accelerate when $W$ is close to $W_{a}$. Therefore, along the transition from a dust universe to a de Sitter one, the expansion starts accelerating, as expected. Close to this transition point the sound velocity reaches its maximum value $c_{s}^{2}=1 / 9$, as discussed in section VII

\section{THE HUBBLE PARAMETER}

As discussed in the preceding sections, the MBI Lagrangian appears as a interesting model for the matter content of the universe, providing an explanation for the accelerated expansion as well as for structure formation. Clearly, in the cosmological setting, among all solutions displayed in the phase plane of figure 4 we must concentrate in the domain characterized by the inequality $\Sigma^{2} / 2<W<\Sigma^{2}$.

To compare the predictions of the model to observational data on the background dynamics, such as the redshift-distance relation, we need to know the evolution of the Hubble parameter. For this sake, the energy density has to be expressed in terms of the scale factor. This is accomplished by solving the energy conservation equation (27), which is conveniently expressed in terms of $W$. Using (22) and after a few manipulations, we get

$$
-3 \frac{\dot{a}}{a}=\dot{W}\left(\frac{1}{2} \frac{1}{\Sigma^{2}-W}-\frac{2}{\Sigma^{2}-2 W}\right) .
$$

Integrating the above equation, in the desired range of $W$, we obtain

$$
\Sigma \sqrt{8 C} a^{-3}=\frac{2 W-\Sigma^{2}}{\sqrt{\Sigma^{2}-W}}
$$

where $C$ is a (conveniently defined) integration constant. This relation is easily inverted to get

$$
W=\left[\frac{1}{2}+C a^{-6}\left(\sqrt{1+C^{-1} a^{6}}-1\right)\right] \Sigma^{2},
$$

were the sign of the root was chosen such that $W \rightarrow$ $\Sigma^{2}$ when $a \rightarrow 0$, to have a dust behavior in the young universe. Inserting (36) in (22) and using (35), we finally get

$$
\rho=A \Sigma^{2} \sqrt{\frac{2}{C}} \frac{\left[\frac{1}{2}+C a^{-6}\left(\sqrt{1+C^{-1} a^{6}}-1\right)\right]^{3 / 2}}{\sqrt{1+C^{-1} a^{6}}-1} a^{3} .
$$

It is easy to see that, in the limit $a \rightarrow 0, \rho \rightarrow A \Sigma^{2} \sqrt{8 C} a^{-3}$, and the scalaron density scales as CDM. On the other hand, in the limit $a \rightarrow \infty$, the fundamental state (24) is reached. Thus, as expected, this model behaves as CDM at early times and goes to a cosmological constant-like state in the future, being a candidate for quartessence. In fact, this asymptotic behavior occurs in any equation of state that intersects the $p=-\rho$ line [13].

In a realistic cosmological situation one has to consider also the baryons and the radiation components. Defining $\Omega_{i}=\rho_{i} / \rho_{\text {crit }}=8 \pi G \rho_{i} /\left(3 H^{2}\right)$, with $H=\dot{a} / a$, the Friedmann equation is given by

$$
H^{2}=\frac{8 \pi G}{3} \sum_{i} \rho_{i}-\frac{k}{a^{2}}=H_{0}^{2}\left(\sum \Omega_{i}^{0} \frac{\rho_{i}}{\rho_{i}^{0}}+\Omega_{k}^{0} a^{-2}\right),
$$

where $\Omega_{k}^{0}=-k / H_{0}^{2}$, the subscript $i$ denotes each component, and the knot means that the quantity is computed at the present value of the scale factor $a=a_{0}:=1$. For baryons $\rho_{b} / \rho_{b}^{0}=a^{-3}$, while for radiation $\rho_{r} / \rho_{r}^{0}=a^{-4}$. The energy density in the scalaron field (37) shall be denoted as $\rho_{s}$. From expression (37) it is clear that $\rho_{s} / \rho_{s}^{0}$ is a function only of $C$ and $a$. Therefore, as in the BI case, although the Lagrangian (21) has two free parameters, the observables depend upon a single free parameter. As the parameter $C$ lacks a direct physical interpretation, it is convenient to define the quantity

$$
R:=\frac{\rho_{\mathrm{v}}}{\rho_{s}^{0}}=\frac{A \Sigma^{2}}{2 \rho_{s}^{0}}
$$

which, as can be seen from (37), depends only on $C$. The parameter $R$ is a measure of how close the present energy density in the scalaron field is to its final "vacuum" value. In other words it is a measure of how close our universe is to its final de Sitter state. From the definition above, it is clear that $0 \leq R \leq 1$. The explicit form of $C(R)$ is

$$
C=\frac{1}{8} \frac{(2 f-1)^{2}}{1-f}
$$

with

$$
f=\frac{\left(3 \sqrt{3} R+\sqrt{1+27 R^{2}}\right)^{2 / 3}-1}{R\left(3 \sqrt{3} R+\sqrt{1+27 R^{2}}\right)^{1 / 3}} \frac{\sqrt{3}}{6} .
$$


Defining $\rho_{s} / \rho_{s}^{0}:=g(a, R)$, were $g$ is obtained from (37) together with (39) and (40), the Hubble function will be given by

$$
\frac{H^{2}}{H_{0}^{2}}=\Omega_{s}^{0} g(a, R)+\Omega_{b}^{0} a^{-3}+\Omega_{r}^{0} a^{-4}+\Omega_{k}^{0} a^{-2},
$$

where $\Omega_{k}^{0}=1-\left(\Omega_{s}^{0}+\Omega_{b}^{0}+\Omega_{r}^{0}\right)$ and $H_{0}$ is the Hubble constant. In the next section we shall use recent data on the distance-redshift relation determined from type Ia supernova observations to set constraints on $R$.

\section{CONSTRAINTS FROM TYPE IA SUPERNOVAE}

Type Ia supernovae (SNIa) observations have now become a standard tool for cosmology. Empirical studies show that these supernovae can be good standard candles after light curve calibration (see e.g. refs [50, 51]). The first compelling evidence from a single experiment for the accelerated expansion of the universe was indeed based on SNIa data 1]. Larger data sets including higher redshift supernova are currently available 3, 4, 52]. These new data makes a stronger case for the current accelerated expansion, since, as expected, a deceleration is detected for higher redshift supernovae, weakening the concerns that the reported acceleration could be due to systematic effects.

Here we shall use some of these recent data to check whether the MBI model can provide a good fit to SNIa observations and to set constraints on the parameter $R$ (eq. 38). For this sake, a sample of 253 SNIa was compiled from references [3] and [52] which provide tables with the redshift $z, \log D_{L}$, and its variance $\sigma_{\log D_{L}}^{2}$ for each supernovae. Here $D_{L}$ is the luminosity distance times the Hubble constant, which, in the flat case, is given by

$$
D_{L}=d_{L} H_{0}=(1+z) H_{0} \int_{0}^{z} \frac{d z^{\prime}}{H\left(z^{\prime}\right)} .
$$

The Hubble parameter $H(z)$ is obtained from equation (eq. 41) with $z=a^{-1}-1$.

Since observations of anisotropies in the CMBR indicate that the Universe is nearly flat $\left(\Omega_{k} \ll 1\right)$, the analysis that follows will be restricted to the zero curvature case. Also, at the redshifts probed by SNIa data, the radiation contribution to the Hubble parameter (eq. 41) is negligible. Therefore, we set $\Omega_{s}^{0}+\Omega_{b}^{0}=1$. We also fix the baryon density parameter at $\Omega_{b}=0.04$, in agreement with the observed abundances of light elements 53, 54 together the Hubble Space Telescope key project result [55]. After these choices, $D_{L}$ becomes solely a function of the MBI parameter $R$.

Following [3] and [52] we discard local supernovae with $z<0.01$, because the peculiar motion contribution to $z$ is too high, and those with high host extinction, $A_{V}>0.5$, which could cause a strong bias in the determination of $D_{L}$. After these cuts, we end up with a sample of 194 SNIa extending up to $z=1.75$. Despite eliminating low redshift SNIa, peculiar motions affect the measurement of $z$ at all redshifts, causing a scatter around the value given by the bulk cosmic expansion. This is taken into account by including an uncertainty in $z$ which is propagated into the luminosity distance and is summed in quadrature with the observational uncertainty in $D_{L}$. Therefore, the Chi-squared is defined by:

$$
\chi^{2}=\sum_{i=1}^{194} \frac{\left[\log \left(D_{L}^{O b s}\left(z_{i}\right)\right)-\log \left(D_{L}^{T h}\left(z_{i}\right)\right)\right]^{2}}{\sigma_{\log \left(D_{L}\left(z_{i}\right)\right)}^{2}+\left(\left.\frac{\partial \log D_{L}^{T h}}{\partial z}\right|_{z_{i}} \sigma_{z}\right)^{2}}
$$

where the theoretical prediction is computed from (42) together with (41) and the observational values are given in the tables of [3] and [52]. Here we assume a velocity dispersion of $\sigma_{v}=500 \mathrm{Km} / \mathrm{s}\left(\mathrm{such}\right.$ that $\sigma_{z} \simeq \sigma_{v} / c \simeq$ $\left.1.7 \times 10^{-5}\right)$.

With the above described assumptions and data set, the best fit value is $R=0.713$ which corresponds to $\chi^{2}=$ 198.0. Therefore the Chi-squared per effective degrees of freedom (193 in this case) is given by $\chi_{d o f}^{2}=1.026$. The MBI model provides a fit as good as for both the $\Lambda \mathrm{CDM}\left(\chi_{d o f}^{2}=1.029\right)$ and Born-Infeld $\left(\chi_{\text {dof }}^{2}=1.020\right)$ models, which also have a single free parameter. The reduced Chi-squared is also very close to other models of quartessence with two free parameters, such as the Generalized Chaplygin Gas $\left(\chi_{d o f}^{2}=1.025\right)$, Exponential Quartessence $\left(\chi_{d o f}^{2}=1.021\right)$, and Logarithmic Quartessence $\left(\chi_{d o f}^{2}=1.023\right)$ [56]. However, as pointed out before, these models are incompatible with largescale structure data for adiabatic perturbations [17, 18].

Defining the likelihood by $\mathcal{L}(R) \propto e^{-\chi^{2} / 2}$, we have checked that the probability distribution of $R$ is very close to a Gaussian, with less than $1 \%$ deviation. At $1 \sigma(68.3 \%)$ confidence level we have $R=0.713 \pm 0.029$. Thus, the current SNIa data is able to limit the MBI free parameter to a rather narrow interval. This makes the model readily testable by using different observables. If the values of $R$ are incompatible to the ones obtained from SNIa, the model must be discarded.

\section{CONCLUDING REMARKS}

Inspired in the Born-Infeld theory for a scalar field, we have introduced a new model for dark-matter and dark-energy unification, the EBI Lagrangian (16). We have developed a systematic study of a subclass of this Lagrangian, namely the MBI model, for which the trajectories in the phase space of homogeneous cosmologies were computed. It was shown that the trajectories of cosmological relevance end up asymptotically in a de Sitter state.

A new feature of this model is the fact that the equation of state changes concavity. Therefore, the sound 
velocity is maximal at an earlier epoch, contrary to the previously considered convex quartessence models, where $c_{s}$ is greater at present [18]. This could solve the known problems associated with the power spectrum of adiabatic quartessence [17]. Therefore, this model has advantages over other generalizations of the Born-Infeld Lagrangian [15, 49].

We have obtained an explicit form for the Hubble function, which depends on a single free parameter (once the $\Omega_{i}^{0}$ are fixed), expressed in terms of the ratio of the fundamental state and current scalaron densities $\left(R=\rho_{\mathrm{v}} / \rho_{s}^{0}\right)$. We applied this result to obtain the luminosity distanceredshift relation and used recent type Ia supernovae data to place constraints on $R$ assuming a flat universe. The model provides a good fit to the data, giving a similar $\chi_{d o f}^{2}$ as the $\Lambda$ CDM model - with the same number of degrees of freedom - and other models of quartessence.

We find that $R=0.713 \pm 0.029$, at $68.3 \%$ confidence. Therefore, contrary to other quartessence models, whose allowed parameter values are highly degenerate 16, 41, 42, 56], this model is tightly constrained by current data. Once $R$ is fixed, the model provides clear predictions for other cosmological observables, such as the matter and CMBR power spectrum.

The results reported in this paper motivate further studies of the EBI model, both to perform a detailed study of its dynamics in the general case, as well as to obtain observational constraints on the model. In particular, in what regards the MBI case, a more complete observational analysis should be performed, both to check if the model withstands to other observables and to improve the statistical analysis. For example, we could have marginalized over the Hubble constant and baryon fraction, instead of fixing these parameters to their bestfitting values. We could also study the effects of spacial curvature on the determination of the parameters. It is fundamental to investigate the formation of cosmic structures in this scenario, both in the linear and nonlinear regimes. Nonetheless, the results of this work indicate that the model here introduced may be a viable candidate for quartessence.

As a final remark, let us recall that in the quartessence model there is an effective transformation of dark-matter into dark-energy and that, in the homogeneous case, around $96 \%$ of the cosmic matter ends up in the de Sitter fundamental state. This brings us to a comment by $\mathrm{S}$. Carrol, stating that "[the de Sitter solution] represents the only natural stable solution for cosmology, and one of the outstanding problems is why we don't find ourselves living there" [57]. In the MBI model, and in the quartessence scenario in general, the answer to this question is: we are indeed heading there!

\section{Acknowledgments}

We thank the participants of the "Pequeno Seminário" for useful comments and suggestions. MM acknowledges Raul Abramo, Patrick Greene, Francesca Perrotta, and
Jaílson Alcaniz for useful comments and suggestions. MM is grateful to the organizers of the COSMO'04 meeting and wishes to thank the hospitality of the Theoretical Astrophysics Group at Fermilab. MN is partially supported by the Brazilian research agencies $\mathrm{CNPq}$ and FAPERJ. MM acknowledges financial support from FAPERJ and CNPq. LSW is partially supported by FUJB. CAR is partially supported by $\mathrm{CNPq}$.

\section{APPENDIX: SPECULATIONS ON THE POSSIBLE ROLE OF SCALARONS IN THE EARLY UNIVERSE}

In this section, we conjecture about the possible role of scalarons in solving two fundamental problems of cosmology: the homogenization of the universe and the issue of initial conditions. First, let us point out that scalarons propagate as tachyons. Indeed, from section III the propagation of the scalarons is provided by $k_{\mu} k_{\nu} g_{e f f}^{\mu \nu}=0$, where the effective metric is given by equation (8). Thus, in the domain $\Sigma^{2}>W>\Sigma^{2} / 2$ we find, from (9), that the scalarons propagate outside the local light cone

$$
k_{\mu} k_{\nu} g^{\mu \nu}=\frac{\Sigma^{4}}{\Sigma^{2}-2 W} \frac{\left(k_{\mu} \nabla^{\mu} \varphi\right)^{2}}{L^{2}}<0 .
$$

For the present energies in the cosmos, the interaction of the scalaron with normal matter must be very weak, otherwise it would not be dark or it would have already been detected in the laboratory. However, the coupling of the scalaron to ordinary matter could be important in the early universe. One may wonder if the superluminal propagation of scalarons could have an important role in the homogenization of the universe.

Another interesting point relates to the initial conditions. One of the possible natural initial configurations is that the scalar field be produced in the state of maximal "kinetic term", i.e., with maximal space-time fluctuations. If on one hand it may be hard to understand how could the universe be "prepared" in a state of infinite density, on the other hand it is perhaps natural to accept that the scalaron was "prepared" in such a state of maximal kinetic term $\nabla_{\mu} \varphi \nabla^{\mu} \varphi=\Sigma^{2}$, which in turn leads to a divergent $\rho$. If radiation is coupled to the scalaron in the early universe, the temperature could be very high due to the thermal equilibrium with the scalaron. A coupling to the electromagnetic field could be introduced in a simple way considering a gauge interaction with a complex scalar field. Notice that the results of the previous sections do not change if we consider a complex scalar field instead of a real one. For a discussion of couplings to other fields see e.g. refs. [23, 58].

Therefore, the MBI model might provide some hints about the early universe, being responsible for the extremely high densities as well as for the cosmic homogenization. These speculations will be subject of further study. 
[1] S. Perlmutter et al., Nature, 391, 51 (1998); A. G. Riess et al., Astron. J. 116, 1009 (1998); S. Perlmutter et al., Astrophys. J. 517, 565 (1999).

[2] P. De Bernardis et al., Nature 404, 955 (2000); S. Hanany et al., Astrophys. J. 545, L5 (2000).

[3] B. J. Barris et al., Astrophys. J. 602, 571 (2004).

[4] A. G. Riess et al., Astrophys. J. 607, 665 (2004).

[5] L. M. Krauss, Astrophys. J. 604, 481 (2004); L. M. Krauss and B. Chaboyer, Science 299, 5603, 65 (2003).

[6] D. N. Spergel et al., Astrophys. J. Suppl. 148, 175 (2003); C. L. Bennett et al., Astrophys. J. 583, 1 (2003).

[7] M. Tegmark, A. J. S. Hamilton, and Y. Xu, Mon. Not. R. Astron. Soc. 335, 887 (2002); M. Tegmark et al. (the SDSS collaboration), Phys. Rev. D 69, 103501 (2004); M. Tegmark et al. (the SDSS collaboration), Astrophys. J. 606, 702 (2004).

[8] S. W. Allen, P. R. W. Schmidt, H. Ebeling, A. C. Fabian, and L. van Speybroeck, Mon. Not. Roy. Astron. Soc. 353, 457 (2004); S. W. Allen, P. R. W. Schmidt, and A. C. Fabian, Mon. Not. Roy. Astron. Soc. 334, L11 (2002).

[9] P. Fosalba, E. Gaztañaga, and F. Castander, Astrophys. J. 597, L89 (2003); R. Scranton et al., astro-ph/0307335; M. R. Nolta et al., Astrophys. J. 608, 10 (2004); N. Afshordi, Y.-Sh. Loh, and M. A. Strauss, Phys. Rev. D 69, 083524 (2004); S. Boughn and R. Crittenden, Nature 427, 45 (2004); P. Fosalba and E. Gaztañaga, Mon. Not. Roy. Astron. Soc. 350, L37 (2004); E. Gaztanaga, M. Manera, and T. Multamaki, astro-ph/0407022

[10] For reviews, see, e.g., G. Bertone, D. Hooper, and J. Silk, Phys. Rept. 405, 279 (2005); K. Griest and M. Kamionkowski, Phys. Rep. 333, 167 (2000); M. S. Turner, Phys. Rep. 197, 67 (1990); G. G. Raffelt, Phys. Rep. 198, 1 (1990).

[11] For reviews see, for instance, M. S. Turner, Phys. Rep. 333, 619 (2000); V. Sahni and A. A. Starobinsky, IJMPD 9, 373 (2000); S. M. Carrol, Living Reviews in Relativity 4, 1 (2001); P. J. E. Peebles and B. Ratra, Rev. Mod. Phys. 75, 559 (2003).

[12] A. Kamenshchik, U. Moschella, and V. Pasquier, Phys. Lett. B 511, 265 (2001).

[13] M. Makler, Gravitational Dynamics of Structure Formation in the Universe, $\mathrm{PhD}$ Thesis, Brazilian Center for Research in Physics (2001).

[14] N. Bilic, G. B. Tupper, R. D. Viollier, Phys. Lett. B 535, 17 (2002).

[15] M. C. Bento, O. Bertolami, and A. A. Sen, Phys. Rev. D 66, 043507 (2002).

[16] M. Makler, S. Q. Oliveira, and I. Waga, Phys. Rev. D 68, 123521 (2003).

[17] H. Sandvik, M. Tegmark, M. Zaldarriaga, and I. Waga, Phys. Rev. D 69, 123524 (2004).

[18] R. R. R. Reis, M. Makler, and I. Waga, Class. Quantum Grav. 22, 353 (2005).

[19] L. J. Hall, J. Lykken, and S. Weinberg, Phys. Rev. D 27, 2359 (1983); E. Cremmer, S. Ferrara, L. Girardello, and A. Van Proeyen, Nucl. Phys. B 212, 413 (1983); S. K. Soni and H. A. Weldon, Phys. Lett. B 126, 215 (1983); M. Visser, Aspects of Supersymmetry Breaking, PhD Thesis, University of California, Berkeley (1984), available at http://www.physics.wustl.edu/ visser/thesis.ps
[20] D. Gross and E. Witten, Nucl. Phys. B 277, 1 (1986); J. Polchinski, Superstrings (Cambridge University Press, Cambridge, 1998), Vol. II, Chap. 12.

[21] L. P. Chimento, M. Forte, and R. Lazkoz, astro-ph/0407288; L. P. Chimento, Phys. Rev. D 69, 123517 (2004).

[22] D. Kutasov, M. Marino, and G. W. Moore, JHEP 0010, 045 (2000); K. Li and E. Witten, Phys. Rev. D 48, 853 (1993); A. Sen, JHEP 9910, 008 (1999).

[23] N. Arkani-Hamed, H.-Ch. Cheng, M. A. Luty, and S. Mukohyama, JHEP 05, 074 (2004).

[24] C. Armendariz-Picon, T. Damour, and V. F. Mukhanov, Phys. Lett. B 458, 209 (1999).

[25] J. Garriga and V. F. Mukhanov, Phys. Lett. B 458, 219 (1999).

[26] C. Armendariz-Picon, V. Mukhanov, and P. J. Steinhardt, Phys. Rev. D 63, 103510 (2001); C ArmendarizPicon, V. Mukhanov, and P. J. Steinhardt, Phys. Rev. Lett. 85, 4438 (2000); T. Chiba, T. Okabe, and M. Yamaguchi, Phys. Rev. D 62, 023511 (2000); T. Chiba, Phys. Rev. D 66, 063514 (2002).

[27] A. Sen, JHEP 04, 48 (2002); G. W. Gibbons, Phys. Lett. B 537, 1 (2002); T. Padmanabhan and T. R. Choudhury, Phys. Rev. D 66, 081301(R) (2002).

[28] M. Novello, S. E. Perez Bergliaffa, and J.M. Salim, Phys. Rev. D 69, 127301 (2004).

[29] R. J. Scherrer, Phys. Rev. Lett. 93, 011301 (2004).

[30] Y. Choquet-Bruhat, C. de Witt-Morette, and M. DillardBleick, Analysis, Manifolds and Physics, North-Holland, New York, 1977, p. 455; see also J. Hadamard, Leçons sur la propagation des ondes et les équations de l'hydrodynamique, Hermann, Paris, 1903.

[31] M. Visser, In: Artificial Black Holes, M. Novello, M. Visser, and G. Volovik, Eds., p. 1, World Scientific, Singapore (2002).

[32] M. Novello, V. A. De Lorenci, J. M. Salim, and R. Klippert, Phys. Rev. D 61, 045001 (2000).

[33] M. Novello and S. E. Perez Bergliaffa, in Proceedings of the Xth Brazilian School of Gravitation and Cosmology, M. Novello and S. E. Perez Bergliaffa eds., AIP Conf. Proc. 668, 288 (2003), gr-qc/0302052.

[34] G. W. Gibbons, Rev. Mex. Fis. 49S1, 19 (2003), hep-th/0106059.

[35] M. Born and L. Infeld, Proc. Roy. Soc. A 144, 425 (1934).

[36] M. Novello, In: Artificial Black Holes, M. Novello, M. Visser, and G. Volovik, Eds., p. 267, World Scientific, Singapore (2002).

[37] S. Chaplygin, Sci. Mem. Moscow Univ. Math. Phys. 21, 1 (1904).

[38] W. Heisenberg, Zeitschrift fur Physik 113, 61 (1939).

[39] D. Bazeia and R. Jackiw, Ann. Phys. 270, 246 (1998); R. Jackiw and A. P. Polychronakos, Commun. Math. Phys. 207, 107 (1999); M. Bordemann, J. Hoppe, Phys. Lett. B 317, 315 (1993).

[40] R. Bean and O. Dore, Phys. Rev. D 68, 023515 (2003); D. Carturan and F. Finelli, Phys. Rev. D 68, 103501 (2003); J. V. Cunha, J. S. Alcaniz, and J. A. S. Lima, Phys. Rev. D 69, 083501 (2004); T. Multamaki, M. Manera, and E. Gaztanaga, Phys. Rev. D 69, 023004 (2004).

[41] M. Makler, S. Q. Oliveira, and I. Waga, Phys. Lett. B 555, 1 (2003). 
[42] R. Colistete Jr, J. C. Fabris, S. V. B. Gonçalves, and P. E. de Souza, Int. J. Mod. Phys. D 13, 669 (2004); L. M. G. Beca, P. P. Avelino, J. P. M. de Carvalho, and C. J. A. P. Martins, Phys. Rev. D 67, 101301(R) (2003); A. Dev, D. Jain, and J. S. Alcaniz, Astron. \& Astrophys. 417, 847 (2004); O. Bertolami, A. A. Sen, S. Sen, and P. T. Silva, Mon. Not. Roy. Astron. Soc. 353, 329 (2004).

[43] L. Amendola, F. Finelli, C. Burigana, and D. Carturan, JCAP 0307, 005 (2003).

[44] R. R. R. Reis, I. Waga, M. O. Calvão, and S. E. Jorás, Phys. Rev. D 68, 061302(R) (2003).

[45] R. R. R. Reis, M. Makler, and I. Waga, Phys. Rev. D 69, 101301(R) (2004).

[46] Z.-H. Zhu, Astron. Astrophys. 423, 421 (2004).

[47] F. Perrotta, S. Matarrese, and M. Torki, Phys. Rev. D 70, 121304(R) (2004).

[48] H. Kodama and M. Sasaki, Prog. Theor. Phys. Suppl. 78, 1 (1984).
[49] L. R. W. Abramo, private comunication.

[50] S. Perlmutter and B. P. Schmidt, In Supernovae \& Gamma Ray Bursts, K. Weiler, Ed., Springer, Lecture Notes in Physics (in press), astro-ph/0303428.

[51] R. A. Knop et al., Astrophys. J. 598, 102 (2003).

[52] J. L. Tonry et al., Astrophys. J. 594, 1 (2003).

[53] S. Burles, K. M. Nollett, and M. S. Turner, Astrophys. J. 552, L1 (2001).

[54] D. Kirkman, D. Tytler, N. Suzuki, J. M. O'Meara, and D. Lubin, Astrophys. J., Suppl. Ser. 149, 1 (2003).

[55] W. Freedman et al., Astrophys. J. 553, 47 (2001).

[56] M. Makler, L. S. Werneck, D. Alencar, and M. O. Calvão, in preparation.

[57] S. M. Carroll, hep-th/0011110.

[58] M. Fierz, Helv. Phys. Acta. 12, 3 (1939); M. Fierz and W. Pauli, Proc. Roy. Soc. Lond. A 173, 211 (1939). 